Euskal ikerketen aldizkaria | Revue d'études basques |

Revista de estudios vascos | Basque studies review

$5 \mid 2000$

Numéro V

\title{
Baxe-Nabarre erresumako herri biltzarreak Izturitzen 1779 eta 1782an
}

\author{
Xipri Arbelbide
}

\section{OpenEdition}

Journals

Édition électronique

URL : http://journals.openedition.org/lapurdum/1336

DOI : 10.4000/lapurdum.1336

ISSN : 1965-0655

Éditeur

IKER

Édition imprimée

Date de publication : 1 octobre 2000

Pagination : 323-333

ISBN : 2-84127-161-7

ISSN : $1273-3830$

Référence électronique

Xipri Arbelbide, "Baxe-Nabarre erresumako herri biltzarreak Izturitzen 1779 eta 1782an », Lapurdum

[Linean], 5 | 2000, Sarean emana----an 01 juin 2009, kontsultatu 01 juillet 2020. URL : http://

journals.openedition.org/lapurdum/1336 ; DOI : https://doi.org/10.4000/lapurdum.1336 


\title{
Baxe-Nabarre erresumako herri biltzarreak Izturitzen 1779 eta $1782 a n$
}

\author{
Xipri Arbelbide
}

Izturitzeko herriko biltzarren berri badakigu 1779ko agorrilaren 13tik. Egun hortan hasten da eskutan daukagun lehen liburua. Nabarreko Erresuma bazen oraino, nahiz botere gehienak kenduak izanak zizkion emeki emeki Frantziako erregeak. Legebiltzarrea Paben biltzen zen.

Erresuma ttipi horren berezitasunetarik bat zen, herritarrak biltzen zirela bere herriko erabakien hartzeko. Ez zen oraiko demokrazia, normalki etxeko jaunek baizik ez baitzezaketen parte har biltzarre hortan, bainan orduan bestetan egiten zenari konparatu behar zaio hemengo joka moldea eta ez orduan hemen egiten zena orai bestetan egiten denari.

Gizona gizon izanez, orduan ere patar guziak ez ziren ordoki eta hain zuzen herriko liburu zaharrenaren lehen orrialdeak kondatzen dauku nola, orduko « demokrazia » hori ere, ez zabilala beharko zukeen bezela : herriak hartzeko orde erabakiak, zenbeitek eskuratua zuten botere guzia, demokrazia eskernio batzu baizik ez eginez. Izturitzen ere gaizki jokatzen ote ziren, ez dugu jakinen ez baitugu dokumentu zaharragorik. Dena den hona Pabeko Parlamentutik 1779ko agorrilaren 13 an igorri zuten paperra.

\section{1- 3.8.1779: Pabeko dekeretua}

\section{Arrest du parlement de Navarre du 13 aoust 1779}

Sur ce qui a été représenté à la cour par le procureur/Géneral du Roy, que la forme des assemblées de Communautés/de son ressort, a toujours mérité de sa part une attention particulière./quelle avait lieu d'espérer que des arrest de Règlement rendus en divers/tems notamment celui du 3 février 1766 produiroient les avantages qu'elle/s'était proposés, que néanmoins une Expérience journalière avoit appris que/cet arrest n'avoit pas son exécution; qu'il lui étoit même revenu. que des/gens mal intentionnés abusoient de ses dispositions pour extorquer des/signatures de la part des habitans qui n'avoient pas assisté aux assemblées/en les menaçant, s'ils refusoient de signer la délibération, hors le lieu/de l'assemblée, de leur 
faire payer la peine portée par le dit arrêt, peine dont ils/obtenoient la décharge, moyennant leur signature, qu'en effet presque toutes/les délibérations générales des communautés qui sont présentées à la cour sont annoncées/comme prises par unité des suffrages et signées par tous les habitans qui ont/sÿu le faire; quelles ne constatent presque jamais le nom de ceux qui sont/présents à l'assemblée et passent pour des délibérations générales des comtes/lors même quelles ne sont prises ni signées que par la moindre partie des/habitans. Qu'enfin il est prétendu que souvent les délibérations sont portées/dans les assemblées toute prêtes à signer, que même on les porte quelques fois maisons par maisons pour avoir des signatures sans qu'il y ait eu aucune assemblée, et comme ces abus ne peuvent que causer de grands/préjudices aux comtés, jeter le trouble et la confusion dans leur/administration les livrer aux intrigues et aux cabales d'un petit nombre/Dhabitants et donner souvent lieu à des procès ruineux pour les $d . / \mathrm{com}^{\text {tes }}$ le prour général a crû qu'il étoit important dy pourvoir.

a ces causes requeroit ordonner $1^{\circ}$ que ledit du mois d'avril 1682 sera/Exécuté selon sa forme et tenuer ce faisant, que dans les assemblées/générales des com tis du ressort la proposition qui devra faire l'objet de la/délibera ${ }^{o n}$ sera faite en l'assemblée par le maire ou premier officier/municipal; qu'après que chacun aura délibéré ; l'arreté seraformélà la pluralité des suffrages, et que l'acte qui sera dressé du tout sera/reçu par le greffier dans les villes et lieux ou il y aura hotel de villelet dans les autres lieux par un des notaires royaux de Larrondissemen.

$2^{\circ}$ Quavant la proposition le greffier de ville ou notaire constatera en tête de la délibération les noms de tous les habitans presens a l'assemblée, qu'ensuite la proposition sera écrite avec lavis de celuy quil'aura faite; ce fait que chaque habitant sera interpellé à son tour et l'avis de chacun deux redigé par écrit à mesure qu'il l'aura donné et que l'acte qui sera dressé dutout sera signé dans l'assemblée et sans déplacer par tous les délibérans qui pourront/et scauront signer.

$3^{\circ}$ qu'il ne sera écrit aucune délibération que sur des registres à ce déstinés sans qu'en aucun cas on puisse en retenir sur feuille volante; /auquel effet toute et chacune des comtés du ressort de la cour seront tenus/de se procurer des registres pour servir au d. usage sy fait n'a été ;/Enjoindre aux Jurats d'y tenir la main chacun endroit soy à peine de Desobeis ${ }^{c e}$.

$4^{\circ}$ Que l'arret du 3 février 1776 sera executé selon sa forme et teneur se faisant/que tous les habitants qui ont droit d'assiter aux assemblées y seront/appelés trois jours a l'avance et seront tenus de sy rendre aux Jours et heures/qui leur seront Indiqués lorsqu'ils seront avertis, comme aussy délibérer chacun/dans son rang et de signer la délibération, avant de sortir de Lassemblée à peine/de trois livres contre chacun des refusans et dilayant, amoins dexcuse legitime/reconnue telle par les officiers municipaux, sans que la d. peine de trois livres puisselêtre remise ny modérée; et seront les contrevenans contrains chacun endroit soy/aupayement, par la voye depignoration et vente des effets pignorés aux formes/ordinaires, nonobstant toutes oppositions reformations et appellations quelconques/sans y préjudicier. Et afin que personne n'en prétende cause d'ignorance, ordner/que larrêt qui Interviendra sera imprimé et publié et affiche par tout 
où besoin/sera et transcrit sur les registres des delibérations de chacune des comtés du ressortià la diligence de ses substitus qui seront tenus dans certifier la cour dans le mois/a peine de suspension de leurs charges. Sur quoy la cour ordonne que ledit/du mois d'avril 1683 Sera Executé Selon sa forme et tenur, ce faisant que dans les/assemblées généralles des comtés du ressort "la proposition qui devra faire Lobjet de la/délibération Sera faite en l'assemblée par le maire ou premier officier municipal ;/quaprès que chacun aura delibéré l'arrette Sera formé a la pluralité des Suffrages/et que l'acte qui sera dressé dutout sera reÿeu par le greffier dans les villes et lieux/où il y aura hotel de ville, et dans les autres lieux par un des nores Royaux/de Larrondissement; qu'avant la proposition, le greffier de ville ou nore consiatera/en tête de la délibération les noms de tous les habitans presens dans lassemblée/qu'ensuite la proposition sera Ecrite avec lavis de celuy qui laura faite/ce fait que chaque habitant sera Interpellé a son tour et lavis/de chacun deux rédigé par écrit, a mesure qu'il l'aura donné ; et quel'acte qui sera dressé du tout sera signé dans Lassemblée, et Sans se déplacer/par tous les habitans qui pourront et sÿauront signer... quil ne Seralécrit aucune délibération que sur de registres à ce déstinés, sans qu'en aucun/cas on puisse en retenir sur feuille volante, auquel effet toutes et chacunes/des comtés du Ressort de la cour seront tenues de se procurer des registres pour/servir au d. usage, sy fait n'a Eté, adjoint aux Jurats dy tenir la main chacun/en droit soy apeine dedesobeissance, ordonne que l'arret du 3 fevr.1776 Sera sera/sera Exécuté Selon Sa forme et teneur, ce faisant que tous les habitants y seront/appelés trois jours a lavance, et qui ont droit d'assister aux assemblées/seront tenus de sy rendre aux Jours et heures qui leur seront Indiqués/Lorsqu'ils seront avertis Comme aussy dy délibérer chacun dans son rang/et de signer ladelibération avant de Sortir de lassemblée, apeine de trois/Livres contre chacun des refusans et deloyaux a moins dexcuse légtime/reconnue telle par les officiers municipaux, sans que lad. peine de trois livres puisse Etre/remise ny modérée; et Seront contrevenans contraints chacun en droit soy au/payements, par la voye de pignoration et vente des Effets pignorés aux formes/ordres, nonobstant toutes oppositions reformations et appellatioans quelconques/Sans y préjudicier, ordonne enfin que le présent arrêt sera Imprimé lû/publié et affiché par tout oubesoin sera, afin que personne nen prétende/cause d'ignorance, et trancrit sur les Registres des deliberations de chacune/des comtes du ressort, ala diligence des substituts dud. proeur général du/Roy, qui seront tenus den certifier la Cour dans le mois apeine de/suspension de leurs charges. Prononcé à Pau en parlement grand chambre/le treze aoust mil sept cent soixante dix neuf, collationné pour Monsieur/le procureur général signé Mounou Greffier.

Dokumentu hunek erakusten dauku :

1- gure herrietan biltzarreak bazirela herriaren harat hunaten eskutan hartzeko.

2- jende batzuk parte hartzen zutela biltzarre horietan. Ez denek beraz. Badakigu bestalde, etxearen jabe izan behar zela biltzarre hortara joaiteko eta etxe bakotxetik gizon bat joaiten zela : nagusi xaharra edo gaztea.

3- 18 garren mende ondarrean lege hori ez zen errespetatua dokumentu honek dioenaz. Gutiengo batek hartzen zituen erabakiak edo batere biltzarrerik egin gabe, edo biltzarrea egiten zen, bainan erabakiak aintzinetik hartuak eta idatziak ziren ; 
baia emaiteaz besterik ez zezaketen egin hor zirenek ; edo oraino idazten zekitenen ikustera joaiten ziren, etxez etxe, batere biltzarrerik egin gabe, paperraren izenpearazteko, mehatxuak eginez gainera izenpetu nahi ez bazuten : nor zen biltzarrearen eta nor ez, biltzarrearen agerian agertzea. Idazten ez zakitenak, ikustea ere ez zuen balio!

Iduriz auziak ere izaiten ziren molde hortan hartu erabakien gatik.

Lehenago ere horrelako zerbeit gertatua zen 1683 eta 1776an jadanik lege zaharra zer zen oroit arazi behar izan zenaz geroz.

Parlamentuak eskatzen du:

1 Biltzarrean parte hartu behar dutenak, hiru egun aintzinetik abisatuak izan diten.

2 Biltzarre horien berri ez dadien edozein paperetan eman baina erregistro batetan : herri bakotxak lehen bai lehen erosi behar du delako erregistroa jadanik egina ez badu. Erregistro hortan idatziko dira :

a) Biltzarre bakotxarentzat partaide guzien izenak.

b) Auzapezak erranen duen Biltzarrearen gaia.

c) Bakotxak bere ikusmoldea emanen du bederazka eta ikusmolde hori idatzia izanen da.

d) Ageria, biltzarrean berean izenpetuko dute izenpetzen dakitenek.

Grefierrak egiten du lan hori : gure zaharrek grefierra deitzen dute oraino herriko etxeko idazkaria. Eta grefierrik ez delarik, notarioak.

e) Hiru liberaren izuna bada. Bainan ez da argi norentzat : biltzarrera etortzen ez direnentzat ? Jalgi aintzin ageria izenpetu nahi ez dutenentzat ? Ala bientzat ?

Ageri horrek ez dauku erraiten Izturitzen biltzarre horiek behar bezala egiten ziren ala ez. Bainan gauza bat argi ateratzen da: Parlamentuak nahi zuen Nabarreko Erresumako lege zaharra errespetatua izan zedien eta biltzarreak demokratikoki iragan ziten, denek parte hartuz, denek beren ikusmoldea emanez.

\section{2- Dekeretua Izturitzen}

Gure lehen erregistro huntako bigarren dokumentua ere biziki interesanta da. Gehienek uste dute apezek eta Elizak zutela morala inposatzen herrian. Apezek zutela manatzen ostatuak hetsiak izan ziten elizkizunak ari zirelarik. Apezek zutela asmatua atseko anjelusetik landa etxean sartu behar zela. Hemen ikusten da batere ez zela hala. Parlamentutik dator manua ostatuak noiz behar diren hetsi eta ideki. Elizak eskaturik menturaz bainan biak akort dira gutienez. Parlamentuak ditu finkatzen gaztiguak, eta ez ttipiak !

Usteko izaiteko zen lehenagoko denboretako girixtino haiek paregabeak zirela? Eta ez ! Haiek ziren ostatuan gelditzen elizan izan beharko zuketeelarik. Usteko zinuten oraikoa dela kargutan direnen ezazolkeria. Eta ez! Ordukotzat jadanik herri gizonek legea aplika arazi behar zutelarik, berek hausten zuten eta zigorren emateko partez batere ez egiten! Hona Pabeko Parlamentutik etorri arresta, 1780ko otsailaren lan. 
Arret du Parlement de Navarre

qui fait defense à tous cabaretiers,/de recevoir, retenir dans leurs maisons/et donner à boire la nuit depuis/Langelus et les jours de dimanches/et fettes pendt les soffices divins, du $1^{\circ}$ fevr $/ 1780$

Sur ce qui a Eté représenté à la cour par le procureur Général du Roy, que/Pour le maintien du bon ordre et en Execution du for et des ordonnances Royaux/Elle a rendu divers arrets Pour la Police des cabarets; qu'ils n'ont pas produit tout/le fruit qu'on avoit lieu d'en attendre : que d'après les mémoires qui lui ont été données, /les d. arrêts ont été ignorés dans une partie des Paroisses du Ressort ou qu'ils y sontloubliés, que la plus part des Jurats loin de tenir la main à leur execution, sont les/premiers à y contrevenir, et au lieu de faire les visites ordonnées et en dresser les procédures/ils authorisent par leurs mauvais Exemples le Scandale et la débauche. A quoi Etant/nécessaire de pourvoir : Requeroit ordonner que for Reglement, ordonnances Royaux/et arrest de la Cour concernant la fréquentation des Cabarets Seront Executés Selon/leur forme et tenur, sous les peines portées par Iceux; en conséq ence faire inhibitions/et deffences à toutes personnes de quelque qualité et condition quelles soient de/boire, et à tous cabaretiers de recevoir, retenir dans leurs maisons et donner à boire/la nuit depuis l'Angelus, et les jours de dimanche et fettes pendt les offices divins,/leur faire pareilles defences de Jouer ni donner à jouer dans les d. Cabarets à aucun/Jeu que ce Soit, à peine de cinquante livres contre chaque contrevenant en ce compris/celle de trois livres douze portée et appliquée par le for le Surplus de la d. peine pécuniaire/applicable pour les deux tiers au fisc et le tiers restant au Dénonciateur; laquelle peine/ne pourra être remise ni être réputée communatoire sous quelque prétexte que ce/puisse être, et Sera payée Solidairement par pignoration et vente des meubles et/Subsidiairement par les autres voix de droit. Enjoindre aux maires Jurats et autres/officiers de police du Ressort de visiter de tems en tems les Cabarets, principalement/pendant les office divins et pendant la nuit le cas y échéant : de dresser procédure/des contreventions et de decerner la $d$. peine de cinquante livres, sans delay ny remise'contre les coupables, à peine contre eux en cas de négligence ou connivence de pareille/peine de cinquante livres applicables comme ci dessus ; a ses fins ordonner qu'il sera/informé d'office ou sur la plainte des dénonciateurs de la négligence ou connivenceldes $d$. officiers de police le cas y echeant par ses Substituts pour au raport etre dit/droit ainsi qu'il appartiendra et afin que personne n'en Ignore; ordonner que/larret qui interviendra Sera lu publié affiché et en registré dans les Sénéchaussées/et Bailliages et transcrits dans les registres des délibons des comtés du Ressort/enjoueindre a Ses Substituts dy tenir la main et d'en certifier la Cour dans le/mois sur quoy la cour faisant droit de la réquisition du Proeur Général/du roy, ordonne que le for reglement, ordonnances Royaux et arrest de la cour concernant la fréquentation des Cabarets Seront Executés selon leur formelet teneur sous les peines portées par iceux, en conséquence fait Inhibition et/deffence à toutes personnes de quelque qualité et conditions quelles soient/deboire et atous Cabaretiers de recevoir retenir dans leurs maisons et donner/à boire la nuit depuis l'Angelus et les Jours de dimanche et fettes pendant les/offices divins, leur fait pareille deffense de jouer ni donner à jouer dans les d./Cabarets à aucun jeu que ce soit, à peine de cinquante livres contre 
chaque/contrevenant en ce compris celle de trois livres douze sols portée et appliquée/par le for le Surplus de la d. peine pécuniaire applicable pour les deux tiers au/fisc et le tiers restant au Dénonciateur laquelle peine ne pourra être remise ny être/réputée comminare Sous quelque prétexte que ce puisse être, et Sera payée/solidairement par pignoration et vente des meubles et subsidiairement/par les autres voix de droit. Enjoueint aux maires, Jurats et autres officiers/de police du ressort de visiter de temps en temps les cabarets, principalement/pendant les offices divins et pendant la nuit le cas y échéant, de dresser/procédure de Contreventions et de decerner la d. peine de Cinquante Livres/Sans delay ny remise contre les Coupables à peine contre eux en cas delnégligence ou connivence de pareille peine de cinquante livres applicable comme/dessus a Cesfins ordonne qu'il sera informé doffice ou sur la plainte des/Dénonciateurs de la négligence ou connivence des d. officiers de policelle cas y échéant par les substituts dud. Proeur général pour au raport/des informations être fait droit ainsi qu'il appartiendra et afin quelpersonne n'en Ignore ordonne que le présent arrêt sera imprimé lu publiélet affiché par tout ou besoin sera et en registré dans les sénéchaussées et/Bailliages et transcrits dans les registres des délibérataons des Com ${ }^{t s} / d u$ ressort. Enjoueint aux Substituts du d. proeur general dy tenir la/main et d'en certifier la Courdans le mois fait à Pau en Parlement/Grand chambre le premier février mil sept cent huitante collationné/pour Monsieur le Procureur Général du Roy signé Bacarere

\section{3- Izturitzeko lehen biltzarrea}

Erregistroaren idekitzeko manua 1779 ko agorrilaren 13 koa zen eta berehala erosi zuten Izturitzen behar zen erregistroa, Parlamentuaren bi arrestaren kopiatzeho zeko. Bainan Izturitzeko biltzarreak ez dira aipu 1782ko urtats arte. Arte hortan biltzarreak izan dira Izturitzen, zerga biltzeko eta urteko juradoen izendatzeko baizik ez bazen ere. Bainan ez dugu horien berririk. Hiru urte pasa eman dute delako erabakiari plegatzen. Hona beraz dekeretuaren arabera egin lehen biltzarrearen berri. Ikusiko da Pabeko manueri jarraikitzen zaizkietela.

L'an mil sept cent Quatre vingts deux et le premier Janvier/apres les divins offices a lendroit de lecolle du lieu disturits/ouj e me suis rendu requis pardevant moy nore Royal/Soussigné présents les témoins bas nommés ont comparu en/assemblée capitulaire convoquée aux formes ordinaires/Estebé me de Bordato, bertrand cadet d'oyhoberro Jurats/du présent lieu, domingo me delissabelar, pierre me/de Candau, pedro $m^{\natural}$ Diriberry, députés du meme présent lieu, $m^{e}$ Guillaume de Pelegrin prour du Roy du Siège de/Lalcadie, Joannes $m^{e}$ Daldave, pedro mettayer de/Sorhouet $J o^{e s} m^{e}$ de Bidart, arnaud me de pochelu, pre/me Diriart, Joes me Detchepare, le mettayer Duhalde, $/ J o^{e s} m^{e}$ de Garat, $J o^{e s} m^{e}$ Durruty, Bernard $m^{e} / d e$ Sorzabal, le mettayer de heguy, Salvat $m^{e}$ Jeune de/Baccardats, Jo ${ }^{e s} m^{e}$ Detchevehere, le mettayer de Sarhi,/françois me Detcheverry, Jo $\mathrm{m}^{e}$ de Larzabal, le $\mathrm{m}^{e} / \mathrm{Duhart}, \mathrm{Jo}^{e s} \mathrm{~m}^{e}$ Damestoy, le $\mathrm{m}^{e}$ Dirigoyen, tristant/me Daguerre, estiene me Daguerrebehere, le me de/Laharrague, tristant Dithurbide faisant pour sa sourlainée Bertrand $m^{e}$ de Carabindeguy Bp $p^{i e} m^{e}$ de Bidegain, le $m^{e}$ de Jeymeteguy, le $m^{e}$ deLaborde,/gratian $m^{e}$ Doyhanburu, le me Dansarola, leme/Doyharisbehere, 
arnaud me de Guelariteguy, le mdeBordato faisant pour la messe Detchegaray, françois $m^{e}$ de Salaberry, Louis me deBergeret, $/ J^{e s}$ me de Celhaito, et de Recalde, Estiene $m^{e} /$ Docquerteguy, pasco $m^{e}$ de Sagarzet, pedro $m^{e}$ de/Garacoits, le $m^{e}$ Durcuroy, la messe Dithurralde, $J o^{e s} m^{e}$ delissagaray den haut, michel/ $m^{e}$ delissagaray de bas, pierre $m^{e}$ Dinta,lle mettayer de harispe, arnaud mettayer de/serorateguy, Crouseilles mettayer de Guichonateguy, /le $m^{e}$ Destebendeguy, le $m^{e}$ de pethansouhi/Joes $m^{e}$ de mendiburu, le $m^{e}$ de Carricaburu, /le $m^{e}$ de Cauliteguy, Bernard mettayer de Bordagaray, le mettayer Detchessoury, le $m^{e}$ de/pascorenia, le $m^{e}$ de nabillaguileteguy, le $m^{e}$ /engagiste Dilaindeguy, pierre $m^{e}$ de Chapital/Jo ${ }^{e s}$ $m^{e}$ de Joaneteguy, le $m^{e}$ de Castondeguy et pierre $m^{e}$ de Basterretche, tous habitans du/présent lieu, lesquels unanimement ont arresté/que le Sr tomieu Continuera sa régence aux gages/ordinaires de cent cinquante Livres par an qui a/comancẻ le ler dexbre dernier, à condition qu'il/sera exact à tenir les Ecoles à l'endroit usité scavoir/dans la présente malon Commune; arretté aussi/que les registres de la communauté demeureront/en ses mains pour y recourir lors que le cas escherra et ceux qui scavent écrire ont signé : Pelegrin, Ste marie, Dyharse, Garra, Lagrenade, dargois,/Lagrenade, Constantin, Campagne nore Royal. J. $b^{t e}$.

Bi ohar biltzarre huntaz :

1- Eskolan egiten da. Non zen eskola hori ? Gehienetan kalustrape gaineko gelan. Hala ote zen Izturitzen ere ? Ez da nihon ageri.

Eskola bazen jadanik Izturitzen, errient berarekin, Tomieu jauna bere errientgoan « segituko » duela diotenaz gain. Orduko eskoletan, gutik ikasten zuten idazten, zenbeitek irakurtzen eta denek, gogoz, katixima.

Baldintza bat emaiten diote errientari : eskola egin dezan ohiko tokian, biltzarrea egin den gelan. Zergatik tokiaren baldintza hori ? Bere etxean egiten ote zuen. eskola, edo beste nonbeit... denbora berean, eskola utzirik, beste zerbeit ere egiten zuela?

2- Ainitz partaide bada : hiruetan hogoi (61). Bainan gauza harrigarria, Nabarreko legearen arabera nagusiek baizik ez zuten parte hartzen ahal biltzarrean. Parte hartzeko eskubiderik ez zuten. Hameka badira hemen, \% 18 : bederatzi etxetiar eta bi emazte. Eta ez dira ondarrera emanak, bainan besteekin nahasteka, nagusi eta etxetiar, gizon eta emazte, elgarren artean diferentziarik ez balitz bezala.

Handik eta zenbeit urteren buruan Frantziako iraultza etorriko delarik « suffrage universel » aipatuko dute bai ! Bainan gizonentzat bakarrik eta doi bat diru. dutenentzat : hiru egunen soldataren heineko zerga pagatu beharko da bozkatzeko eskubidea izaiteko.

Emazte baten partaidetza etxetiarrena baino harrigarriagokoa da oraino. Ithurraldeko eta Etxegaraiko etxekandereak hor ditugu gizonen artean ! Biga baizik ez, erranen dute zenbeitek ! Frantzian, 1945 urtea igurikatu behar izan da emaztek bozkatzeko eskubide xoila izateko.

Bestalde bi gizon badira bi emazteren izenean emanak : hor izan beharko zuketen beraz beste bi emaztek. Horietarik batek, andreiak, bere anaia gazteagoa igorri du. Bi horiek ez ote litezke menturatu gizonen artean agertzera ? Dena den, bi gizon horiek, bi emazte haien ordezkari baizik ez dira. « Emaztearen izenean » dira hor : zinez emazteak zeukan boterea. 
Garai beretan, lau emazte ikusi ditugu Heletako Biltzarre batetan, dotzena erdi bat Mehainen.

Etxetiarrak hor dira kasik beti Heletan eta dotzena erdi bat urtez lau juradoetarik bat etxetiarra zen. Bi mende pasa igurikatu beharko da gero etxetiar baten berriz ikusteko Heletako kontseiluan.

Bost hautetsi badira : hiru deputatu, hauen lana delarik zergen biltzea herrian. $\mathrm{Bi} \mathrm{jurado} \mathrm{bestalde,} \mathrm{horiek} \mathrm{direlarik} \mathrm{herriko} \mathrm{kontseilua.} \mathrm{Heletan} \mathrm{lau} \mathrm{baziren} \mathrm{bainan}$ deputaturik ez da agertzen, hemen hiru badirelarik.

Ez da errana partaide bakotxa nola mintzatu den, Pabeko erabakiak eskatzen zuen bezela ; arrazoin xinple baten gatik : denak akort dira.

Guillaume de Pelegrin, sortzez Garruzekoa zen eta Sokobiako alabarekin ezkondua. Prokuradorea, hor idatzia den bezala, lege gizona Arberuako alkadian : Heleta, Donostiri, Meharin, Donamartiri, Izturitz eta Aiherrak egiten zuten Arberuako herri elkartea. Herri nagusia Donamartiri zen eta hor zen alkade edo jujea, bere prokuradore eta uxerrarekin.

Pabeko arrastak eskatu bezela, erret notario batek, J.B. Campagne Bastidakoak egin du idazkari lana.

\section{4- Izturitzeko $3^{\circ}$ biltzarrea}

1782ko martxoaren 12an izan zen biltzarre bat. Bi juradoek (Esteven Floren Bordattoko nagusiak eta Bertrand d'Tharse Oyhoberrokoak) jakin arazi zuten zerga biltzeko izendatuak izanak ziren lau deputatuek (Harizmendy Etxartekoa, Durkiet Elizbelharrekoa, Pedro Iriberrikoa, eta Issouribehere Candaukoak) beren kargua uzten zutelako berria uxerraren bidez emana zutela : arazoak bazituzten jendeekin delako zergaren biltzeko. Demisione hori onarturik, juradoen gain utzia izan zen zergaren biltzea martxoaren 12ko biltzarrean.

Bainan afera ez zen hortan gelditu eta Pabeko arresta hitzez hitz segitzen zuen biltzarre bat izan zen 1782ko azaroaren 9an.;

L'an mil sept cens quatre vingts deux et le neuf novembre/vers les dix heures du matin au lieu disturitz et maison Comune/en assemblée capitulaire convoquée aux formes ordinaires/tenue par $s^{\text {s }}$ pierre Lagrenade Me Diriart et Saubat/Etchepare $m^{e}$ Durcudoy Jurats du présent Lieu dans laquelle/se sont trouvés Me Guillaume de Pelegrin mérin et Prour/du Roy du siège de Lalcadie $S^{r}$ de Soccobie, $S^{r}$ martin de Ste marie $m^{e}$ en chirurgie, Joannes $m^{e}$ de Joanateguy,/Saubat $m^{e}$ Jeune de Baccardats, Bertrand $m^{e}$ Dilaguindeguy,/Raymon Constantin cordonnier, arnaud $m^{e}$ de Jeymeteguy,/Joannes Lamarque $m^{e}$ de Bicharchouriteguy, françois $m^{i} /$ Detcheverry, Louis me de Bergeret, pierre $m^{e}$ de/Candau, Joannes $m^{e}$ de Celhaito, pedro me de Damestoy gratian héritier duhalde, Bernard $m^{e}$ de Sorzabal,/Jacques $m^{e}$ dalsuya, françois $m^{e}$ de Salaberry/Joannes $m^{e}$ de Touloumont, arnaud $m^{e}$ de Guelariteguy, pasco $m^{e}$ de pascoenia, Joannes $\mathrm{m}^{e} / d e$ mendiburu, estebe $m^{e}$ de Bordato, Bertrand $/ m^{e}$ de Carabindeguy, Joannes me Diriberry, Joes $m^{e}$ ancien de Sagarzet, Gratian $m^{e}$ Doyhanburu,/pierre $m^{e}$ de Chapital, Baptiste $m^{\varepsilon}$ de Chouriateguy,/pedro Duhalde mettayer de Sorhouet, pierre $m^{e}$ inta,/Joannes harismendy $m^{e}$ Detchart, tristan $m^{e}$ asarola,/Joannes $m^{e}$ 
Jeune Detchepare, Joannes $/ m^{e}$ de Larzebal, pasco $m^{e}$ Jeune de Sagarzet, pierre/me de Laharrague, pierre cadet de Castondeguy, etienne $m^{*} /$ Daguerrebehere, pierre $m^{e}$ de Carricaburu. dans laquelle assemblée les $d S^{\text {th }}$ Jurats ont représenté lexploit/dune requette aEux signifiée le 31 8bre der par Darsapalo/huissier présentée à la Cour du parlement le 26 du même/mois par les $S^{\text {ss }}$ harismendy, Durquiet, pedro $m^{2}$ Diriberry/et pierre Dissouribehere anciens députés du présent lieu/dans laquelle ils se plaignent de la délibération prise par la présente Communauté, qui supprime les députés/concluant casser lad. délibération avec des deffences/tant aux Jurats et habitans den prendre de semblables/alavenir, ce faisant enjoindre aux Jurats et habitans de/procéder dans 8ne conformément a lancien usage a la nomination de quatre députés pour la répartition/des Impositions Royalles, charges locales et la confection/de Rolles ainsi que pour assister aux assemblées généralles/du païs, laquelle requette aété appée d'un soit montrée (?)/apartie et au procureur général, demandant les dits $/ S^{r s}$ Jurats de délibérer sur le parti àprendre dans/la Circonstance, Lavis du $S^{r}$ de Lagrenade Jurat/est que la délibération du 12 mars dernier qui contient/la suppression dont les $d . S^{\text {ss }}$ harismendy et consorts se plaignent/soit executée suivant sa forme et teneur, et qu'a ces fins il soit nommé un syndic pour la deffendre aux/dépans delacomunauté, nommant de son cotté pour tel/syndic le Sr de Pelegrin, lavis dud. S Durcudoy,/autre Jurat est que l'usage ancien soit suivi et qu'à/ces fins il soit nommé quatre députés en conformitéldes conclusions prises dans la d. requette, Lavis dud./S Pelegrin est que la délibérataion dud. jour/12 mars soit exécutée, attendu que les députés navoient/aucun pouvoir de rien régaller dans la Comunautélqu'après le Consentement de celle cy pour imposer telle/ou telle chose ou pour ne point limposer, quils étoient/dailleurs onéreux à la Comunauté puisqu'ils avoient/quinze Livres de Gages annuellement sans aucun travail/ny service, aucun ne sachant ny lire ny Ecrire/et tout le travail devant être confié au Scribe/qui dailleurs était payé, ces députés ne pretant/pas non plus aucun serment, ny ne pouvant/certifier leurs opérations, et aux fins de la deffense/de lad. délibération nomme le d. Sr de Ste marie/pour Sindic, et que les fraix, dépans, et avances/quil faira en cette occasion seront suportées par/la Comunauté au Marc la Livre, Lavis du S de Ste marie est dememe, et Nomme led. Sr Lagrenade/pour sindic, Lavis du dBernard Sr de Sorzabal est/aussi dememe, et nomme ledSr de Ste marie pour/sindicq, le Sr de Baccardats est de Lavis dud. Sr Durcudoy/Jurat, Bertrand Me Dilaindeguy est de/Lavis dud. Sr de pelegrin meme pour le sindic, le/me de Larsabal est de Lavis dud. $S^{r}$ Durcudoy/Jurat, le me de Bergeret est aussi dumeme avis, le/me de Laharrague est dumeme avis, le $m^{e}$ de Carricaburu/idem, le $m^{e}$ jeune de Sagarzet Idem, le me ancien de/Joanateguy Idem, le $m^{e}$ de Celhaito Idem, le $m^{e} /$ Damestoy idem, le $m^{e}$ de pascoenia Idem, le $m^{e} /$ Daguerrebehere Idem, le cadet de Castondeguy/est de lavis du d. Se. de Pelegrin, leme de Salaberry/est de lavis dud. SrDurcudoy, le me Dalcuya Idem, /le me de Guelariteguy Idem, le me de mendiburu est/de lavis du Sr. de Pelegrin, leme de Bordato Idem, /le me de Carabindeguy Idem, leme de Touloumont/Idem, le me Detcheverry delavis du $S^{7}$ Durcudoy, le me Doyhambourou Idem, le me de Chapital Idem, /le mettayer de Sohouet dit qu'il n'a pas de voix sur le/sujet dont il est question et quil n'est comparu a la/présente assemblée que pour obeir a lavertissement, le me Dinta est de lavis dud. Se 
Durcudoy, le me/Dassarola est dumeme avis, le me jeune Detcheparre/est de Lavis dud. Se de Pelegrin, Lhéritier Duhaldelest de Lavis du Sr Durcudoy, et les suffrages ayant/été ensuite comptés, il été vérifié que celuy du Sr/Durcudoy a prévalu etdautant que Lassemblée/n'est pas daccord concernant ceux qui doivent et peuvent être nommés deputés, elle a renvoyé/cette opération a un autre Jour, elle a délibéré/dailleurs quil sera procédé par les Jurats à pignoration contre les habitans deffaillants a la/presente assemblée pour leur faire payer les peines/qu'ils ont encourues, ceux qui savent écrire ont/signé

Lagrenade, Pelegrin, Ste marie, Garra, Mendibourou,,

Campagne $N^{\text {re }}$ Royal.

Biltzarre honen aintzinekoan (1785-3-12) Izturitzek erabaki zuen deputatu kargua kentzea, deusetako ez zelakotz eta gostatzen. Kargutan ziren deputatuek afera auzitan eman zuten eta Parlamentuak manatu zuen deputatuak berriz izendatzea.

Azaroko biltzarrean ikusten da nola atxikitzen duten kondu Pabetik etorri manuetaz biltzarrea egiteko moldean. Partaide guzien izenak agertzen dira, biltranzarrearen gaia, bakotxaren ikusmoldea gai hortaz, bederazka. Eta ikusten da ere biltzarrera etorri ez direnak gaztigatuak direla : " pignoration » hitz horrek erran nahi du zerbeit bahitzen zutela dirua izan arte. Sorhueteko etxetiarra zigorraren beldurrez etorria zen, gaiaz ez zuelarik ikusmolderik. Sagarzetetik nagusi zaharra eta gaztea biak hor ziren. Aldi hontan etxetiar bakar bat baizik ez da, Sorhouet, bainan pentsatzeko da Constantin zapataina ere ez zela etxeko nagusi : ez dugu jakin ahal izan zein etxetarik etorria zen.

Deputatuen lanaz gehiago jakiten dugu : Arberuako biltzarrean parte hartzen zuten, herriaren izenean.

Etxeko nagusi andana bat falta da, 41 partaide baizik ez denaz gain, Iehen aldian 61 zirelarik. Hor ez direnak zigortuak izanen dira. 41 horietarik $32 \mathrm{k}$ baizik ez dute beren ikusmoldea emaiten.

1789an etorriko zen Frantziako Iraultza eta biltzarre horiek desagertuko dira. Moda xaharreko azken biltzarrea 1789 ko irailaren 7 an egin zen Izturitzen. 


\section{Résumé :}

Les assemblées de village en Basse Navarre au Xvil' siècle : Isturitz en 1782

Le registre d'Isturitz nous donne le texte du décret du Parlement de Navarre du 13 août 1779 expliquant la raison pour laquelle il demande la tenue de ces registres : dans certaines paroisses on ne tenait plus d'assemblées capitulaires, ou bien elles ne se déroulaient pas conformément à la coutume, contrairement à deux décrets antérieurs, avril 1683 et 3 février 1776.

Le décret de 1779 décrit en détail la façon dont ces assemblées devaient se dérouler.

Pour le cas d'Isturitz, nous voyons que le registre ne sert d'abord qu'à copier plusieurs arrêts du Parlement de Navarre, dont celui du $1^{\circ}$ février 1780 , qui interdit aux cabaretiers d'ouvrir leurs maisons la nuit et pendant les offices religieux.

Il fallut attendre le I janvier 1782, soit trois ans, pour que le décret fût pleinement appliqué : c'est la première assemblée dont nous ayons un compte rendu. Cette assemblée forte de 61 participants décide à l'unanimité de reconduire $M^{r}$ Tomieu comme régent du village et fixe les conditions du contrat.

Les deux assemblées suivantes dont il est rendu compte datent du 12 mars et du 9 novembre 1782. Nous donnons le texte concernant cette dernière assemblée. Le 12 mars, les habitants d'Isturitz avaient décidé de supprimer la charge de " députés », jugés inutiles et coûteux. Suite à cette décision, les députés en fonction avaient porté plainte et le Parlement de Pau imposa à Isturitz de nommer des députés. L'assemblée du 9 novembre rend compte de la décision du parlement. Nous voyons chaque participant donner son avis, comme le demandait le décret du 13 août 1779. Il y a moins de participants, 41, et les absents seront sanctionnés.

A ces deux assemblées ont pris part des gens qui n'auraient pas dû le faire: des métayers et des femmes. Normalement, seul le chef de famille propriétaire avait droit d'entrée à l'assemblée. 